\title{
Adaptation de la mesure potentiométrique à l'estimation en continu de la teneur en cuivre de solutions aqueuses présentant de fortes variations physico-chimiques Adaptation of potentiometric measurements to continuous estimation of copper in aqueous solutions with fluctuating physicochemical parameters
}

\author{
É. Tisserand, P. Schweitzer, P. Tisserand et J. L. Cécile
}

Volume 14, numéro 2, 2001

URI : https://id.erudit.org/iderudit/705415ar

DOI : https://doi.org/10.7202/705415ar

\section{Aller au sommaire du numéro}

\section{Éditeur(s)}

Université du Québec - INRS-Eau, Terre et Environnement (INRS-ETE)

ISSN

0992-7158 (imprimé)

1718-8598 (numérique)

Découvrir la revue

Citer cet article

Tisserand, É., Schweitzer, P., Tisserand, P. \& Cécile, J. L. (2001). Adaptation de la mesure potentiométrique à l'estimation en continu de la teneur en cuivre de solutions aqueuses présentant de fortes variations physico-chimiques. Revue des sciences de l'eau / Journal of Water Science, 14(2), 157-172.

https://doi.org/10.7202/705415ar

\section{Résumé de l'article}

La mesure en continu, sans prélèvement et sous conditions physico-chimiques variables, de la teneur en cuivre d'une solution aqueuse, constitue le cadre de ce travail. La méthode d'estimation proposée est basée sur la potentiométrie sélective à l'aide d'une électrode spécifique au cuivre. Le comportement de l'électrode est modélisé par une loi non linéaire s'inspirant de la relation de Nernst généralisée. Le modèle intègre les paramètres température, conductivité et $\mathrm{pH}$ de la solution. Il rend possible une estimation de la concentration totale de cuivre même en présence de complexations hydroxyles. La phase de modélisation est présentée en détail. Les performances de l'estimateur sont évaluées puis discutées. 


\title{
Adaptation de la mesure potentiométrique à l'estimation en continu de la teneur en cuivre de solutions aqueuses présentant de fortes variations physicochimiques
}

\author{
Adaptation of potentiometric measurements \\ to continuous estimation of copper in aqueous \\ solutions with fluctuating physicochemical \\ parameters
}

\section{E. TISSERAND $^{1 *}$, P. SCHWEITZER ${ }^{1}$, P. TISSERAND $^{1}{ }^{*}$, J.L. CECILE ${ }^{2}$}

Reçu le 4 juillet 2000, accepté le 7 mars 2001**

\section{SUMMARY}

Ion Selective electrodes (ISEs) offer an attractive solution for continuously evaluating the content of certain ionic species in aqueous media. Manufacturers propose a wide range of electrodes specific to heavy metals $\left(\mathrm{Cu}^{2+}\right.$, $\mathrm{Pb}^{2+} \ldots$... Because they eliminate the need for sampling, are of reasonable size and have few electronic parts, ISEs seem highly appropriate for continuous monitoring in urban purification systems.

Measurements obtained by these sensors in controlled media in the laboratory are usually precise, reliable and reproducible. However, it is not so with complex and uncontrolled media. This work falls within the general scope of the continuous measurement of heavy metals in wastewater. More particularly, it is devoted to the description of the behaviour of a copper-selective electrode ( $\left(\mathrm{ISE}_{\mathrm{Cu}}\right)$ in a medium presenting wide physicochemical variations.

Experimental set-up

In order to study ISE behaviour, we developed an experimental platform that allowed us to reproduce in a reactor the physicochemical variations observed in wastewater, particularly with regards to salinity and acidity. The reactor was fitted with a measuring set consisting of five electrodes that measured the following parameters: $\mathrm{pH}$ (ref. integrated $\mathbf{A g} / \mathrm{AgCl}$ ), redox (red), $\operatorname{ISE}_{\mathrm{Cu}}\left(\mathbf{E}_{\mathrm{Cu}}\right)$, temperature $(\mathbf{T})$ and conductivity $(\sigma)$. A computer system carried out the acquisition of the five signals with a 10-second sampling period. The species concentration in the reactor was determined by calculating the weight of the solutions extracted from or injected into the reactor. Control-

Laboratoire d'instrumentation électronique de Nancy 1, Université Henri Poincaré, BP 239, 54506 Vandoeuvre-les-Nancy, France.

2 Groupement pour l'évaluation des mesures et des composants en eau et assainissement, 149, rue Gabriel Péri, 54500 Vandoeuvre-les-Nancy, France.

* Correspondance. E-mail : etienne.tisserand @lien.u-nancy.fr patrick.schweitzer@lien.u-nancy.fr

${ }^{* *}$ Les commentaires seront reçus jusqu'au 29 mars 2002. 
ling the temperature of the system was undertaken using a cryostat. Sequential tests allowed the $\mathrm{pH}$, redox potential and conductivity of the medium to be varied and were carried out by successive injections of different chemical products. The response times of the conductivity probe and of the $\mathrm{pH}$ and redox electrodes are shown here; the short response time of the sensors (20 to 30 s) and the strong correlation between the measured pH and redox are noted.

\section{ISE modelling}

The model used to explain the ISE response is based on a generalization of Nernst's Law that takes into account the temperature and the activity of the free ions $\left(\mathrm{Cu}^{2+}\right)$. Taking into consideration chemical equilibria and mass equations allowed us to link the activity of the free copper ions to the total injected copper concentration $\left.\mathrm{Cu}^{2+}\right|_{\text {tot }}$ and to the $\mathrm{pH}$. Redox, strongly correlated to $\mathrm{pH}$, was ignored in the mathematical model. Since hydroxyl complexation is the major complexation reaction (compared to other copper-binding ligands), the potential measured with the ISE took the following form:

$$
\mathbf{E}_{\mathrm{cu}}=\mathbf{b}_{0}+\mathbf{b}_{1} \mathbf{T} \cdot \log \left[\frac{\gamma_{2}\left|\mathbf{C u}^{2}\right|_{\text {tot }}}{1+\mathbf{b}_{2} \gamma_{2} 10^{\mathrm{pH}}+\mathbf{b}_{3} \gamma_{2} 10^{2 \mathrm{pH}}}+\mathbf{b}_{4}\right]
$$

$b_{i}(1<i<4)$ are the model coefficients.

The activity coefficient $\gamma_{2}$ of the $\mathrm{Cu}^{2+}$ ions was calculated from the ionic strength (I) of the solution, using the Debye-Hückel approximation. Ionic strength was derived from conductivity corrected to $25^{\circ} \mathrm{C}$. In wastewater, the ranges of the physicochemical parameters were as follows: $\mathrm{T}$ from 5 to $35^{\circ} \mathrm{C}$; pH from 4 to 9; $\sigma$ from 500 to $2000 \mathrm{mS} / \mathrm{cm}$; redox from 400 to $-400 \mathrm{mV} / \mathrm{ENH}$; and copper concentrations $\leq 10^{-3} \mathrm{~mol} / \mathrm{dm}^{3}$.

In order to identify the $b_{i}$ coefficients of the model, we established an experimental plan comprising 108 measurement points that covered, with a minimal number of experiments, the ranges of variations of the parameters of influence. A dispersion diagram of measured and modelled values gave a linear adjustment coefficient close to 0.99 and a standard deviation of $8.8 \mathrm{mV}$, which corresponds to a 0.34 decadal standard error in the concentration estimate. With a temperature of $25^{\circ} \mathrm{C}$, the model has a sensitivity of $-26.4 \mathrm{mV} /$ decade, very close to the theoretical slope of an electrode sensitive to divalent ions.

\section{ISE measurement of the copper concentration with large $\mathrm{pH}$ variations}

pH is the parameter which exerts the greatest influence on ISE response, which is why tests simulating copper pollution with large variations of pH were carried out. These tests enabled us to evaluate the performances of the model in terms of the estimation of copper content. Four solutions of total copper concentration equal to $10^{-6}, 10^{-5}, 10^{-4}, 10^{-3} \mathrm{~mol} / \mathrm{dm}^{3}$ respectively, were used. Their temperature was $25^{\circ} \mathrm{C}$ and their conductivity was fixed at approximately $500 \mathrm{mS} / \mathrm{cm}$. We varied the pH of each solution between 4 and 10. For the four tests, we show the estimate of the copper concentration obtained with our model starting from the potential measured by the ISE.

In the case of strong copper pollution $\left(10^{-3} \mathrm{~mol} / \mathrm{dm}^{3}\right)$, the model yields an overestimated concentration below $\mathrm{pH} 7$ with a decadal error of less than 0.5 . Above pH 7, the concentration is underestimated while maintaining a decadal error of less than 0.5. At pH 7, a 0.04-decade minimal error is found. For pollution equal to or less than $10^{-4} \mathrm{~mol} / \mathrm{dm}^{3}$, the model gives good results in an acid or neutral medium with a decadal error usually less than 0.3 . In an alkaline medium, concentration is overestimated. In this case the error 
increases in a roughly linear manner with the $\mathrm{pH}$ and the co-logarithm of copper concentration. From the results of these tests, we defined a valid domain of ISE copper concentration measurement using our model.

In conclusion, the suggested method, although not very accurate, could be used as an indicator of the copper concentration level in wastewater. The ISE-response correction model is currently being tested under operational conditions at a water treatment plant in Nancy-Maxéville (France).

Key-words: water quality control, copper pollution, ISE.

\section{RÉSUMÉ}

La mesure en continu, sans prélèvement et sous conditions physicochimiques variables, de la teneur en cuivre d'une solution aqueuse, constitue le cadre de ce travail. La méthode d'estimation proposée est basée sur la potentiométrie sélective à l'aide d'une électrode spécifique au cuivre. Le comportement de l'électrode est modélisé par une loi non linéaire s'inspirant de la relation de Nernst généralisée. Le modèle intègre les paramètres température, conductivité et $\mathrm{pH}$ de la solution. Il rend possible une estimation de la concentration totale de cuivre même en présence de complexassions hydroxyles.

La phase de modélisation est présentée en détail. Les performances de l'estimateur sont évaluées puis discutées.

Mots clés : contrôle de la qualité de l'eau, pollution par le cuivre, électrode sélective.

\section{1 - INTRODUCTION}

Depuis ces dernières années, les lois sur l'environnement font l'objet d'une réglementation exigeante rendant obligatoire une surveillance accrue de la qualité des effluents urbains et industriels rejetés dans la nature. Le contrôle de la teneur en métaux lourds de ces rejets constitue une priorité compte tenu des impacts néfastes qu'ils peuvent avoir sur les écosystèmes et de la toxicité pour l'être humain.

En réalité, il n'y a pas à l'heure actuelle de solutions bien adaptées à un suivi en continu de la concentration en ions métalliques d'un milieu aqueux complexe et non contrôlé. Les méthodes spectro-spectrophotométriques (KATSUHITO et al., 1994) et ampérométriques (MIZIER, 1995), présentent l'avantage d'être précises et sélectives mais sont lourdes à mettre en œuvre. De plus la nécessité de prélèvement dans le milieu interdit la mesure en continu. Du point de vue pratique, les ISFETS (COBBEN et al., 1992) n'ont pas, à l'heure actuelle, tenu les promesses espérées en théorie. Enfin les biocapteurs, plutôt réservés à des stations d'alertes, ne sont pas discriminatifs par rapport aux métaux lourds et ont un temps de réponse trop long (THOMAS et al., 1996).

La mesure, sans prélèvement ni additif, de la teneur en cuivre d'une solution constitue le cadre de travail. Les conditions physicochimiques sont supposées variables. Nos mesures sont basées sur la potentiométrie sélective au cuivre par électrode spécifique (THOMAS et al., 1996) (ISE : Ion Selective Electrode). Le 
$\mathrm{pH}$, le redox, la température et la conductivité, mesurés conjointement, servent à la correction de la réponse de l'électrode.

La relation de Nernst, généralisée par NICKOLSKI et EISENMAN (SAES DE VITERI et al., 1994), met en évidence la dépendance du potentiel des électrodes spécifiques à la concentration en ions primaires et interférents d'une part, à la force ionique et à la température du milieu, d'autre part. Le modèle de réponse que nous proposons s'appuie sur une loi analogue dans laquelle la force ionique est approchée par la mesure de conductivité de la solution. L'introduction du paramètre $\mathrm{pH}$, qui permet l'estimation de la concentration de cuivre même en présence de complexation, constitue l'originalité de notre modélisation. Nous montrons également que l'introduction du redox n'est pas nécessaire à la modélisation de l'ISE.

La première partie présente l'unité de mesure multiparamètres et la plateforme expérimentale utilisées dans nos campagnes de mesures. Nous décrivons également les protocoles d'élaboration des séquences de test qui sont obtenues par injection de différents produits chimiques.

La deuxième partie est consacrée à la phase de caractérisation et de modélisation de l'électrode ainsi qu'à l'organisation du plan d'expériences ayant servi de base à cette étude.

Les résultats de mesure de concentration en cuivre dans diverses situations sont résumés dans une dernière partie. Nous détaillons plus particulièrement ceux relevés sous de fortes variations de $\mathrm{pH}$.

\section{2 - MATÉRIEL ET MÉTHODE}

\subsection{Plage effective de variations des paramètres}

Dans notre étude, nous avons pris en compte les 4 paramètres physicochimiques de la solution suivant : température $(T)$, conductivité $(\sigma)$, redox (Red) et $\mathrm{pH}$.

Tableau 1 Gammes et précisions des mesures.

Table 1 Range and precision of results.

\begin{tabular}{|c|r|r|c|}
\hline Paramètre & \multicolumn{2}{|c|}{ Gamme de mesure } & Précision \\
\hline Température $\left({ }^{\circ} \mathrm{C}\right)$ & 5 & 35 & \pm 0.1 \\
Conductivité $(\mu \mathrm{S} / \mathrm{cm})$ & 0 & 4000 & $\pm 5 \%$ de la mesure \\
$\mathrm{pH}$ & 3 & 10 & \pm 0.1 unité de $\mathrm{pH}$ \\
Redox $(\mathrm{mV})$ & -2000 & 2000 & $\pm 10 \mathrm{mV}$ \\
$\mathrm{pC}_{\mathrm{Cu}}$ & 6 & 3 & non précisée \\
\hline
\end{tabular}

L'influence de ces paramètres sur la qualité des mesures par ISE (Ion Selective Electrode) est certaine. Le tableau 1 reproduit les gammes de varia- 
tions des quatre paramètres physicochimiques et de la teneur en cuivre susceptibles d'être rencontrées dans les eaux de rejet.

\subsection{Tête de mesure}

La tête est constituée de cinq électrodes ( $\mathrm{pH}$-référence hydrogel $\mathrm{Ag} / \mathrm{AgCl}$ intégrée, Redox, $\left(S E_{\mathrm{Cu}}\right)$, une sonde de température Pt100, une sonde de conductivité quatre pôles. L'ensemble est placé dans un support circulaire de type PVC (figure 1). Le tableau 2 résume les spécifications principales des cinq capteurs.

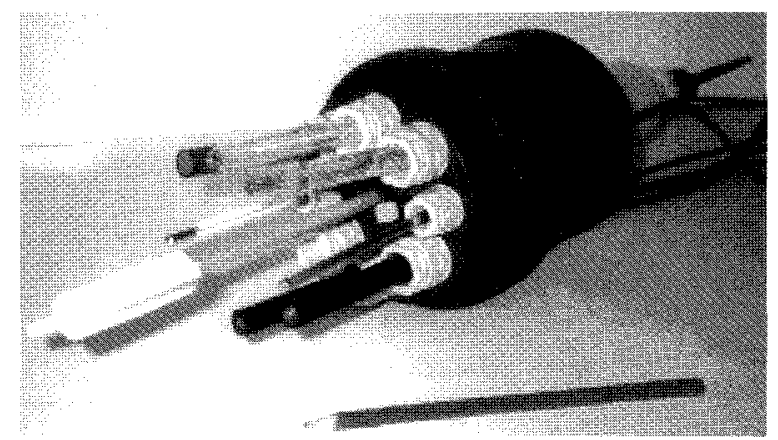

Figure 1 Tête de mesure.

Measuring set.

Tableau 2 Spécifications des capteurs.

Table 2 Probes specifications.

\begin{tabular}{|c|c|c|}
\hline Grandeur & Type & Spécifications \\
\hline Température & Sonde Pt 100 & Précision : $0,05^{\circ} \mathrm{C}$ de 0 à $35^{\circ} \mathrm{C}$ \\
$\mathrm{pH}$ & Ingold (HA 405-DPA-SC-S8/120) & Gamme de mesure : 0 à 14 \\
Redox & Ingold (HA 405-DPA-SC-S8/120) & non précisée \\
Conductivité & CDM210 - Radiometer Analytical & Gamme de mesure : 0 à $2 \mathrm{~S} / \mathrm{cm}$ \\
ISE cuivre & ISE $25 \mathrm{Cu}$ - Radiometer Analytical & Gamme de mesure : $10^{-6}$ à $10^{\circ} \mathrm{mol} / \mathrm{I}$ \\
\hline
\end{tabular}

\subsection{Plate-forme expérimentale}

Afin d'étudier le comportement de la tête de mesure, nous avons développé une plate-forme expérimentale (figure 2) permettant de simuler les variations physicochimiques d'un milieu réel.

L'élément principal est un réacteur contenant les solutions aqueuses sur lequel est insérée la tête de mesure. Celle-ci est équipée de deux tuyaux en Téflon reliés à une pompe et deux mini-vannes permettant d'injecter ou d'extraire des solutions. La concentration des solutions dans le réacteur est obtenue par calcul à partir de pesées des solutions extraites ou injectées. La précision est inférieure à $0,05 \%$ pour les solutions mères, $0,15 \%$ pour les solutions diluée par 10 et $1 \%$ pour les solutions diluées par 100 . 


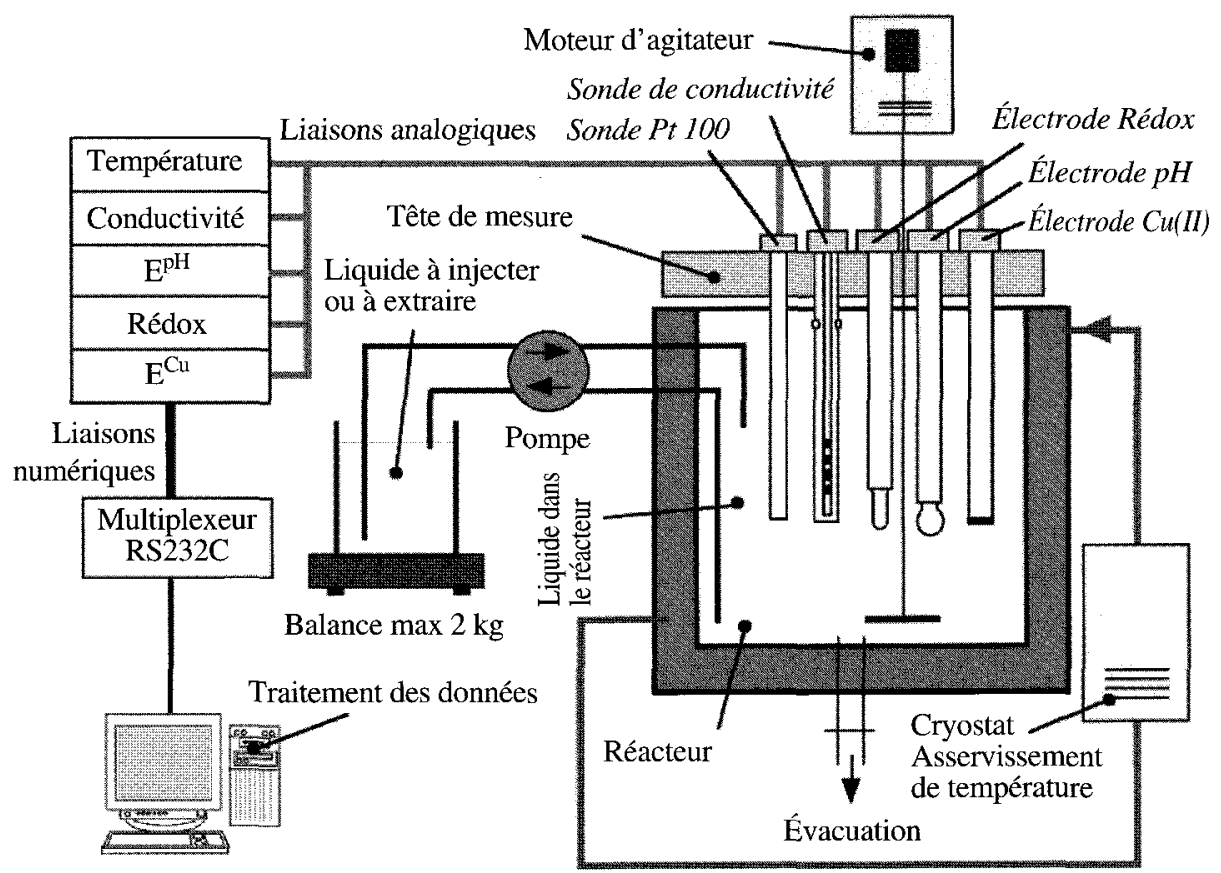

Figure 2 Banc de mesure.

Experimental set-up.

L'asservissement de la température de la solution est obtenue par un cryostat permettant une circulation d'un liquide dans la double enveloppe du réacteur. La qualité de la régulation est de $0,03{ }^{\circ} \mathrm{C} / 72$ heures avec une erreur statique de $0,5^{\circ} \mathrm{C}$.

L'homogénéité des solutions dans le réacteur est obtenue par un agitateur dont la vitesse est ajustée à $140 \mathrm{tr} / \mathrm{mn}$. Cette vitesse est un très bon compromis entre la rapidité d'homogénéisation, l'apparition de vortex, bulles d'air ou de tourbillons et la constante de temps de réponse en température du réacteur.

Tableau 3 Caractéristiques du millivoltmètre et du conductivimètre.

Table 3 Characteristics of the millivoltmeter and conductivity meter.

\begin{tabular}{|c|c|}
\hline Millivoltmètre de laboratoire & Gamme $:-2$ à $2 \mathrm{~V}$. \\
& Résolution $: 0,1 \mathrm{mV}$ \\
PHM 220 Radiometer Analytical & Précision $+1-0,1 \mathrm{mV} . \mathrm{Ze}>2.10^{12} \Omega$ \\
& $\mathrm{le}<0,5 \mathrm{pA}$ à $25^{\circ} \mathrm{C}$ \\
\hline Conductivimètre & Gamme $: 0$ à $4000 \mu \mathrm{S} / \mathrm{cm}$ Résolution : \\
& $1 / 4000$ de la pleine échelle \\
CDM210 Radiometer Analytical & Précision : $0,2 \%$ sur la lecture. \\
\hline
\end{tabular}




\subsection{Instrumentation}

Les différents capteurs sont reliés à un ensemble de conditionneurs chargés de numériser l'information. Un système d'acquisition $(\mathrm{PC})$ et de traitement des données (développé sous HP-VEE), par l'intermédiaire d'un multiplexeur et d'une liaison RS 232C, permet d'échantillonner des données issues de l'ensemble des conditionneurs avec une période de 10 secondes. Les caractéristiques du conductivimètre et du millivoltmètre de laboratoire sont résumées dans le tableau 3.

\section{3 - CONTRÔLE DES PARAMÈTRES PHYSICOCHIMIQUES DE LA SOLUTION}

\subsection{Modes d'action}

Les solutions et moyens permettant de contrôler les paramètres physicochimiques dans le cadre de nos expérimentations sont présentes dans le tableau 4.

Tableau 4 Moyens de contrôle.

Table 4 Control method.

\begin{tabular}{|c|c|c|c|c|}
\hline Paramètres & Température (T) & Conductivité (o) & pH & Redox (Red) \\
\hline $\begin{array}{c}\text { Moyens } \\
\text { et produits } \\
\text { de contrôle }\end{array}$ & Cryostat & $\mathrm{Kcl}$ & $\mathrm{HClO}$ & $\mathrm{Na}_{2} \mathrm{~S}_{2} \mathrm{O}_{4}$ \\
& & $\mathrm{KClO}$ & $\mathrm{KOH}$ & $\mathrm{O}_{2}$ \\
& & $\mathrm{NaCl}$ & $\mathrm{NaOH}$ & \\
\hline
\end{tabular}

\subsection{Interaction dans les contrôles des paramètres physicochimiques}

Une séquence obtenue par des injections successives des différents produits d'action est effectuée. Les évolutions temporelles des mesures des paramètres $\mathrm{pH}, \sigma$ et Red sont présentées en figures 3, 4 et 5 .

D'une manière générale, on constate une réponse rapide des capteurs aux variations du milieu.

La conductivité reste relativement constante après injection du $\mathrm{KClO}_{4} . \mathrm{Ce}$ paramètre peut ainsi être considéré comme indépendant des autres.

II n'en est pas de même pour le redox et le $\mathrm{pH}$ qui présentent entre eux une forte corrélation.

\subsection{Modélisation du comportement de l'ISE}

Plutôt que d'utiliser une modélisation heuristique, nous avons préféré une approche s'appuyant sur la relation de Nernst (1) généralisée aux variations possibles dans notre milieu d'étude. 


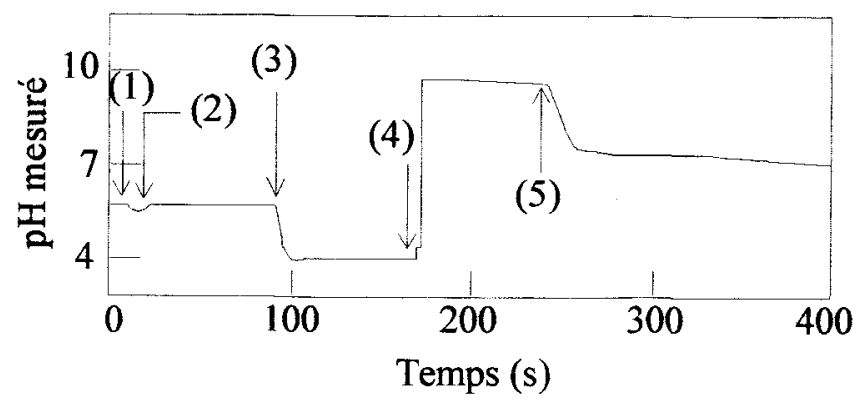

Figure $3 \quad \mathrm{pH}$ en fonction du temps.

$\mathrm{pH}$ as a function of time.

(1) : eau pure ; (2) : injection de $\mathrm{KClO}_{4} ;(3)$ : injection de $\mathrm{HClO}_{4}$; (4) : injection de $\mathrm{KOH}$; (5) : injection de $\mathrm{HClO}_{4}$

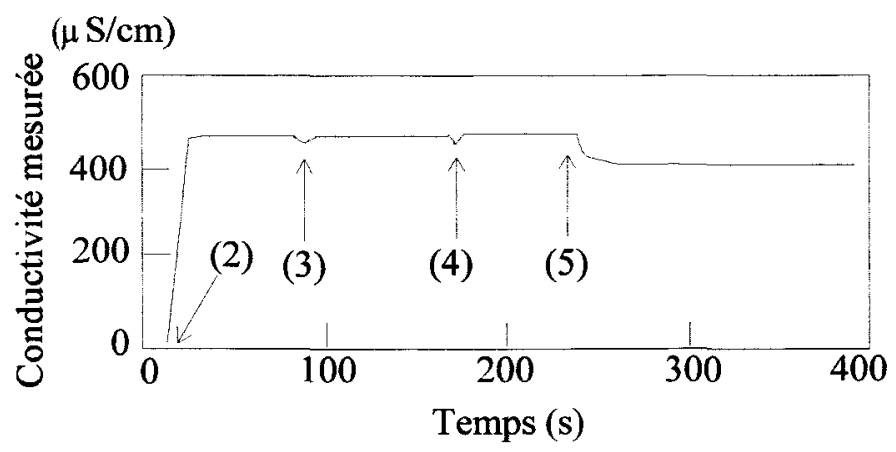

Figure 4 Conductivité en fonction du temps.

Conductivity as a function of time.

(2) : injection de $\mathrm{KClO}_{4} ;(3)$ : injection de $\mathrm{HClO}_{4} ;(4)$ : injection de $\mathrm{KOH} ;(5)$ : injection de $\mathrm{HClO}_{4}$

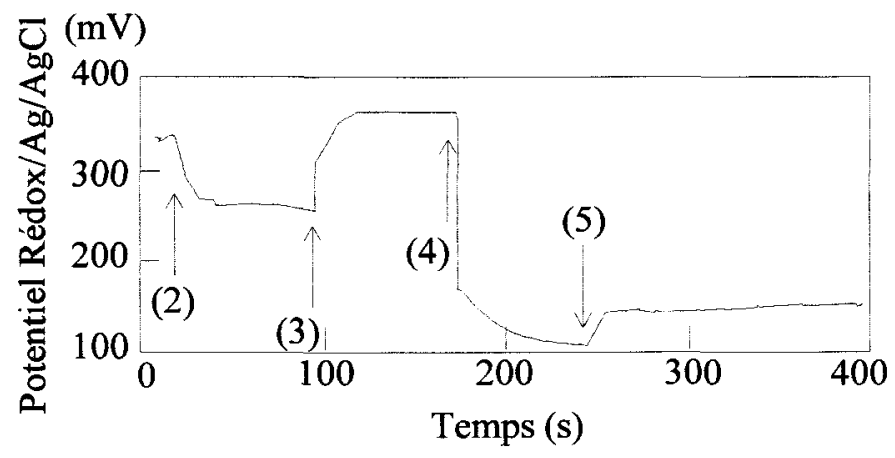

Figure 5 Potentiel redox en fonction du temps.

Redox potential as a function of time.

(2) : injection de $\mathrm{KClO}_{4} ;(3)$ : injection de $\mathrm{HClO}_{4} ;(4)$ : injection de $\mathrm{KOH}$; (5) : injection de $\mathrm{HClO}_{4}$ 
Notation adoptée : IXI est la concentration des espèces $X,(X)$ est l'activité de l'espèce $X$.

$$
E_{C u}=E_{0}+2,3 \frac{R T}{n F} \log \left[\left(C u^{2+}\right)+a_{0}\right]
$$

$E_{C u}$ est le potentiel de l'électrode spécifique de cuivre, T est la température en degré Kelvin.

$\left(\mathrm{Cu}^{2+}\right)$ est l'activité des ions libre de cuivre, $\mathrm{a}_{0}$ est la limite de détection de l'activité.

Nous modifions l'équation (1) afin de faire apparaître les influences de T, du coefficient d'activité et du $\mathrm{pH}$. Le redox, fortement corrélé au $\mathrm{pH}$, est ignoré dans la modélisation. Le coefficient d'activité des ions $\mathrm{Cu}^{2+}$ est déterminé à partir de la force ionique I de la solution, elle même évaluée par l'intermédiaire de la mesure de conductivité.

Nous posons comme hypothèse que les complexes hydroxyles sont majoritaires (par rapport à d'autres composés du cuivre) dans notre milieu suivant l'équation suivante (STELLA et al., 1980) :

$$
\left|\mathrm{Cu}^{2+}\right|_{\text {tot }}=\left|\mathrm{Cu}^{2+}\right|+\left|\mathrm{CuOH} \mathrm{OH}^{+}\right|+\left|\mathrm{Cu}(\mathrm{OH})_{2}\right|
$$

$\mathrm{Cu}^{2+} \mid$ est la concentration des ions libres de cuivre.

À partir des équations d'équilibre, nous exprimons les deux constantes d'équilibre $K_{1}$ et $K_{2}$ en posant comme hypothèse que celles ci ne varient pas dans le domaine de température.

$$
\begin{gathered}
K_{1}=\frac{\left|\mathrm{CuOH}^{+}\right|}{\gamma_{1} \gamma_{2}\left|\mathrm{Cu}^{2+}\right| \mathrm{OH}^{-} \mid} \\
K_{2}=\frac{\left|\mathrm{Cu}(\mathrm{OH})_{2}\right|}{K_{1} \gamma_{1}^{2} \gamma_{2}\left|\mathrm{Cu}^{2+} \| \mathrm{OH}^{-}\right|^{2}}
\end{gathered}
$$

$\gamma_{1}=$ coefficient d'activité des ions $\mathrm{OH}^{-}, \gamma_{2}=$ coefficient d'activité des ions $\mathrm{Cu}^{2+}$

Le coefficient d'activité pour $\mathrm{CuOH}^{+}$et celui de $\mathrm{Cu}(\mathrm{OH})_{2}$ sont pris comme étant égal à 1. En effet, la concentration de $\mathrm{CuOH}^{+}$est très faible et $\mathrm{Cu}(\mathrm{OH})_{2}$ est sous forme neutre et principalement dans une phase séparée.

$\left(\mathrm{Cu}^{2+}\right)$ est effectivement mesurée par l'ISE $\mathrm{Cu}_{\mathrm{u}}$ et représente la quantité de cuivre que nous espérons mesurer.

À partir des équations de masse (2) et des équations d'équilibre, nous exprimons la relation entre $\left|\mathrm{Cu}^{2+}\right|_{\text {tot }}$ et $\left(\mathrm{Cu}^{2+}\right)$

$$
\left|\mathrm{Cu} u^{2+}\right|_{\text {tot }}=\frac{\left(\mathrm{Cu}^{2+}\right)}{\gamma_{2}}\left[1+K_{1} \gamma_{1} \gamma_{2}\left|\mathrm{OH}^{-}\right|+K_{1} K_{2} \gamma_{1}^{2} \gamma_{2}\left|\mathrm{OH}^{-}\right|^{2}\right]
$$

L'équilibre ionique de l'eau est donné par l'équation : $\mathrm{K}_{e}=\left(\mathrm{H}^{+}\right)\left(\mathrm{OH}^{-}\right)$

Avec $\mathrm{pH}=-\log \left(\mathrm{H}^{+}\right)$, nous obtenons : 


$$
\left(\mathrm{Cu}^{2+}\right)=\frac{\left.\gamma_{2} \mathrm{Cu}^{2+}\right|_{\text {tot }}}{1+K_{e} K_{1} \gamma_{2} 10^{p H}+K_{\mathrm{e}}^{2} K_{1} K_{2} \gamma_{2} 10^{20 H}}
$$

A partir des équations (1) et (5) nous pouvons écrire avec

$$
\begin{gathered}
A=\frac{\left|C u^{2+}\right|_{\text {tot }}}{\left[1+b_{2} \gamma_{2} 10^{\rho H}+b_{3} \gamma_{2} 10^{2 P H}\right]}: \\
E_{\text {cu }}=b_{0}+b_{1} T \cdot \log \left(\gamma_{2} A+b_{4}\right)
\end{gathered}
$$

Les coefficients $b_{0}$ à $b_{4}$ sont les paramètres à identifier.

Le coefficient $\gamma_{2}$ est calculé à partir de la force ionique I de la solution selon la loi approchée de Debye-Hückel (7) utilisable dans la gamme de concentration considérée ici :

$$
\gamma_{2} \approx 10^{-2 \sqrt{l}}
$$

Nous proposons alors d'évaluer la force ionique à partir de la mesure de conductivité ramenée à une température de $298 \mathrm{~K}$ (8). Notons $\sigma_{298}$ la valeur de la conductivité corrigée.

$$
I=\frac{1}{2} \sum_{i}\left|C_{i}\right| z_{i}^{2}=\alpha \cdot \sigma_{298}
$$

$\left|C_{i}\right|$ est la concentration de l'espèce $i$ et $z_{i}$ sa valence.

Pour une solution dont la conductivité est fixée par $\mathrm{KClO}_{4}$, le coefficient $\alpha \approx 7,9$ (TISSERAND, 1998).

La correction en température de la mesure de conductivité est donnée par l'équation (9) (TISSERAND, 1998) :

$$
\sigma_{298}=\frac{\sigma+0,55(298-T)}{1+0,017(298-T)}
$$

$\sigma$ est la conductivité mesurée en $\mu \mathrm{S} \cdot \mathrm{cm}^{-1}$ à la température $T$.

\subsection{Plan expérimental}

Pour identifier les coefficients $b_{0}$ à $b_{4}$ de l'équation (6), nous avons élaboré un plan d'expériences couvrant les plages de variation des paramètres d'influence $\left(\mathrm{T}, \sigma, \mathrm{pH}\right.$ et $\left.\mathrm{pC}_{\mathrm{Cu}}\right)$.

Le plan comporte 108 points de mesure résumés dans le tableau 5 . Les valeurs de concentration sont exprimées par le changement de variable $\mathrm{pC}=-\log |\mathrm{C}|$.

Le potentiel redox a été également relevé au cours des 108 expériences. La figure 6 représente ses valeurs en fonction du pH mesuré, ainsi que la répartition des différentes zones de prédominance des espèces proposée par (CHARLOT, 1969). Cette superposition graphique montre que l'hypothèse de complexes hydroxyles majoritaires est vérifiée. II n'y a aucun risque d'obtenir du cuivre sous forme réduite. 
Tableau 5 Plan expérimental.

Table 5 Experimental design.

\begin{tabular}{|c|}
\hline Paramètres \\
\hline Température \\
\hline$-\log (\mathbf{C})$ \\
\hline Conductivité \\
\hline pH \\
\hline
\end{tabular}

Classement de la mesure
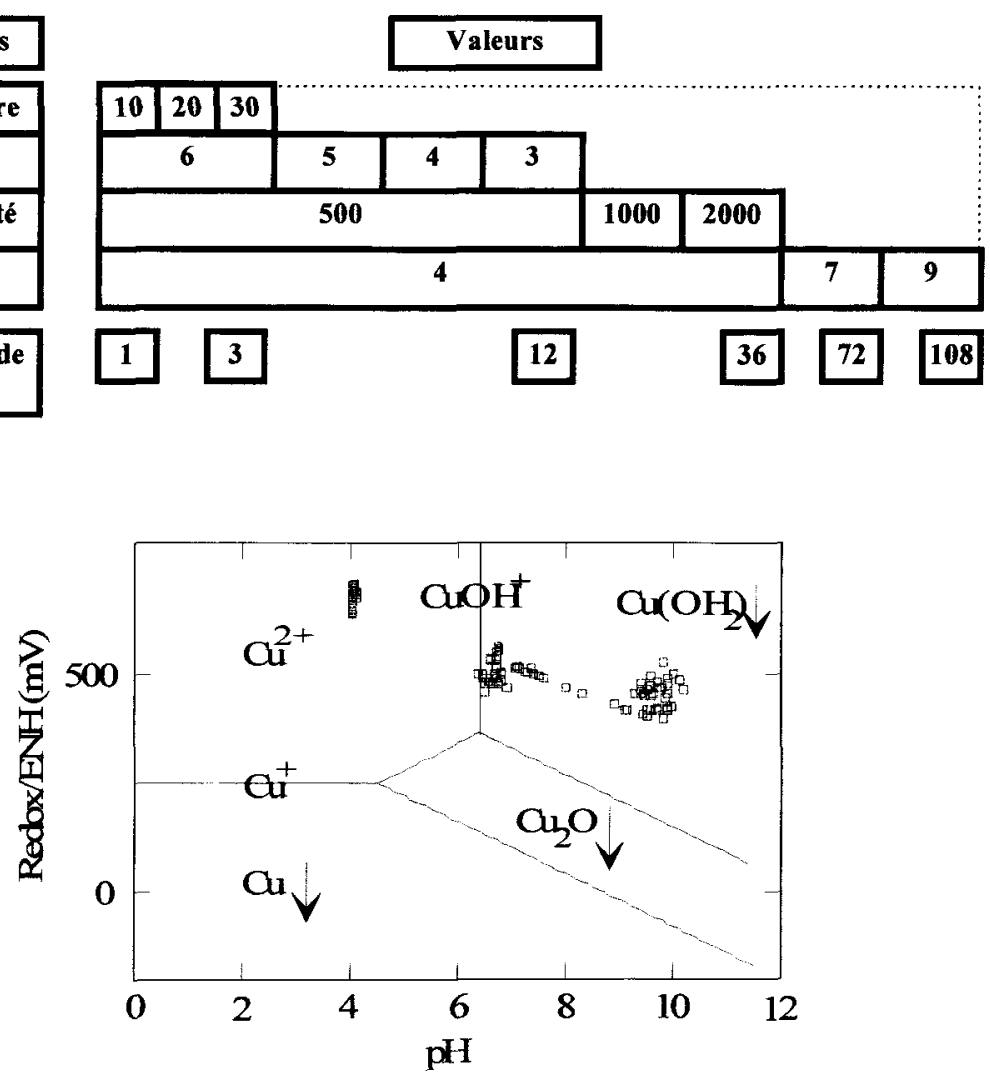

Figure 6 Valeurs mesurées dans le plan $\mathrm{pH}$-redox.

pH-redox values obtained.

\section{4 - ÉTUDE COMPORTEMENTALE}

\subsection{Caractéristiques du modèle}

Les résultats de l'identification des coefficients $b_{0}$ à $b_{4}$ par la méthode des gradients adaptatifs (JUNG, 1986) sont consignés dans le tableau 6.

Tableau 6 Coefficients identifiés.

Table 6 Identified coefficients.

\begin{tabular}{|c|c|c|c|c|}
\hline$b_{0}(\mathrm{mV})$ & $b_{1}\left(\mathrm{mV} \cdot \mathrm{K}^{-1}\right)$ & $\mathrm{b}_{2}\left(\mathrm{~mol} \cdot \mathrm{kg}^{-1}\right)$ & $\mathrm{b}_{3}\left(\mathrm{~mol} \cdot \mathrm{kg}^{-1}\right)$ & $\mathrm{b}_{4}$ \\
\hline $2.36910^{2}$ & $8.8510^{-2}$ & $-1.19710^{-6.8}$ & $1.661710^{-13}$ & $210^{-6}$ \\
\hline
\end{tabular}


Le diagramme de dispersion entre valeurs mesurées et estimées du potentiel $\mathrm{E}_{\mathrm{Cu}}$ est décrit en figure 7 .

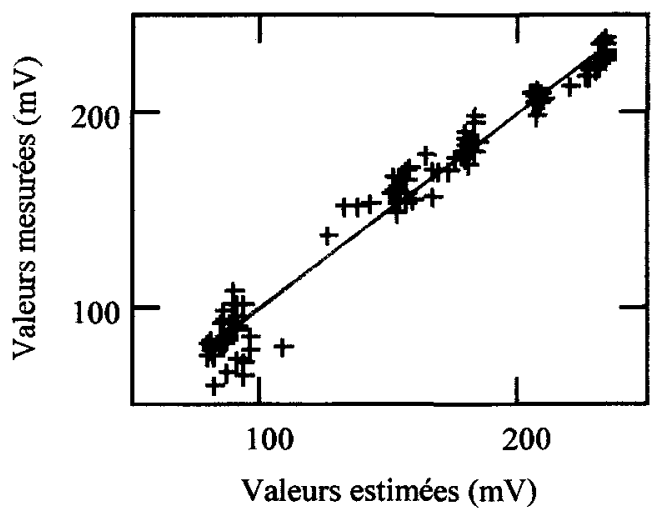

Figure 7 Diagramme de dispersion.

Dispersion diagram.

Le coefficient de corrélation atteint 0.986 et l'écart type entre modèle et mesure se situe à environ $9 \mathrm{mV}$.

La sensibilité du modèle au paramètre $\mathrm{pC}_{\mathrm{Cu}}$ est définie par $\mathrm{S}=\frac{\delta E_{C u}}{\delta p C_{C u}}$.

D'après la relation (6), cette sensibilité peut être évaluée par le produit $b_{1} T$. À $298 \mathrm{~K}$, le modèle présente une sensibilité de $-26,4 \mathrm{mV} /$ décade. Cette valeur est proche de la pente théorique de $-24,58 \mathrm{mV}$ d'une ISE sensible à des ions divalents.

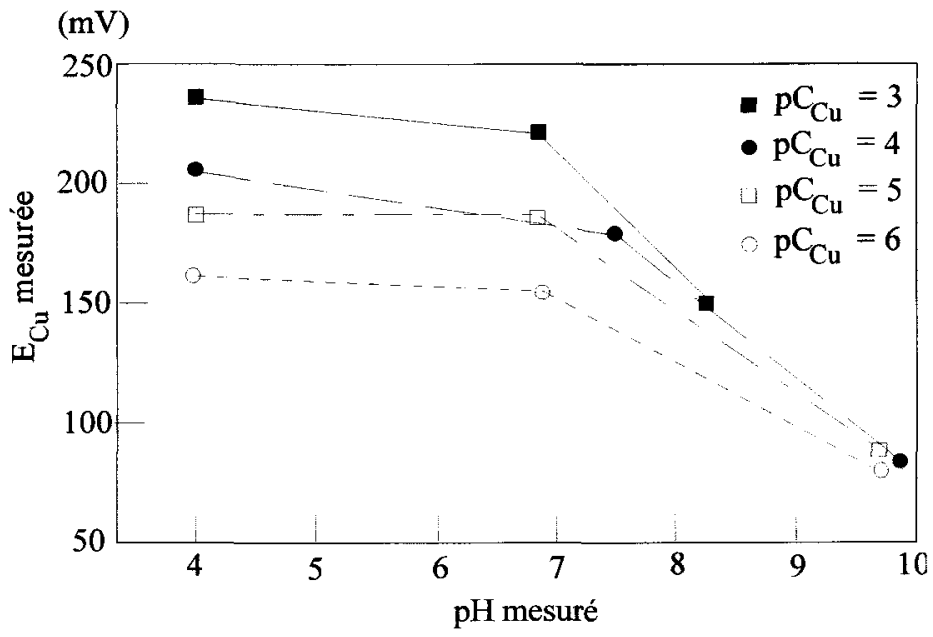

Figure 8 Couples $\left(E_{\mathrm{Cu}}, \mathrm{pH}\right)$ mesurés.

Measured pairs $\left(E_{C u}, p H\right)$. 
L'écart type rapporté à la sensibilité indique une erreur type de 0.34 décade dans l'estimation de la teneur en cuivre de la solution.

L'influence du $\mathrm{pH}$ est traduite dans l'équation (6) par les coefficients $b$, et $b_{3}$. Compte tenu de leurs valeurs, cette influence est significative en milieu basique. La figure 8 , qui représente quelques couples de valeurs mesurées $\left(E_{C u}\right.$ et $\mathrm{pH}$ ), confirme cette constatation.

\section{5 - ESSAIS D'ESTIMATION DE LA TENEUR EN CUIVRE D'UNE SOLUTION PRÉSENTANT DE FORTES VARIATIONS DE PH}

\subsection{Présentation - Conditions de test}

La stabilité du $\mathrm{pH}$ de l'eau dans les sites d'assainissement n'étant pas assurée, nous proposons d'évaluer les performances du modèle d'ISE proposé en terme de précision sur l'estimation de la teneur en cuivre dans le cas de fortes variations de $\mathrm{pH}$. Les conditions opérées lors des tests sont résumées dans le tableau 7.

Tableau 7 Conditions expérimentales.

Table $7 \quad$ Experimental conditions.

\begin{tabular}{|c|c|c|c|c|}
\hline & Essai 1 & Essai 2 & Essai 3 & Essai 4 \\
\hline $\mathrm{pC}$ & 3 & 4 & 5 & 6 \\
$\mathrm{~T}(\mathrm{~K})$ & 298 & 298 & 298 & 298 \\
$\sigma(\mu \mathrm{S} / \mathrm{cm})$ & 500 & 500 & 500 & 500 \\
$\mathrm{pH}$ & 4 à 10 & 4 à 10 & 4 à 10 & 4 à 10 \\
\hline
\end{tabular}

\subsection{Résultats}

Les figures 9 à 12 décrivent l'évolution de l'estimation de la concentration de cuivre en fonction du $\mathrm{pH}$ et de la teneur réellement injectée dans la solution. La valeur de conductivité est ici fixée à $500 \mu \mathrm{S} \cdot \mathrm{cm}^{-1}$.

\subsection{Commentaires}

Dans le cas d'une forte pollution au cuivre $\left(\mathrm{pC}_{\mathrm{Cu}}=3\right.$, figure 9$)$, le modèle restitue une concentration surestimée en dessous de $\mathrm{pH} 7$ avec une erreur inférieure à 0,5 décade. Au-delà, $\mathrm{pC}_{\mathrm{Cu}}$ est sous estimée tout en conservant une erreur inférieure à 0,5 décade. On relève à $\mathrm{pH} 7$ une erreur minimale de 0,04 décade.

Pour des pollutions moyennes à faibles $\left(\mathrm{pC}_{\mathrm{Cu}} \geq 4\right.$, figures 10 à 12), le modèle présente de bons résultats en milieu acide ou neutre avec une erreur toujours inférieure à 0,5 décade et généralement inférieure à 0,3 décade.

En milieu basique la concentration est surestimée. On notera dans ce cas que l'erreur croît de manière approximativement linéaire avec le $\mathrm{pH}$ et la valeur de $\mathrm{pC}_{\mathrm{Cu}}$. 


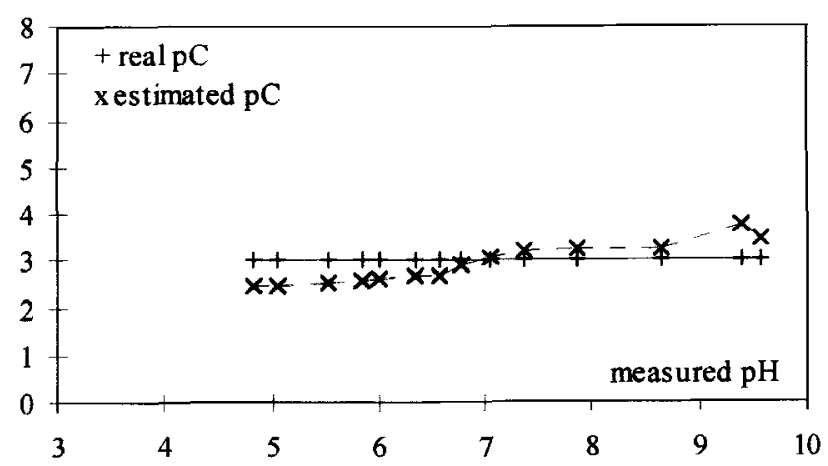

Figure $9 \quad \mathrm{pC}$ estimé dans le test 1. $p C$ estimate, test 1.

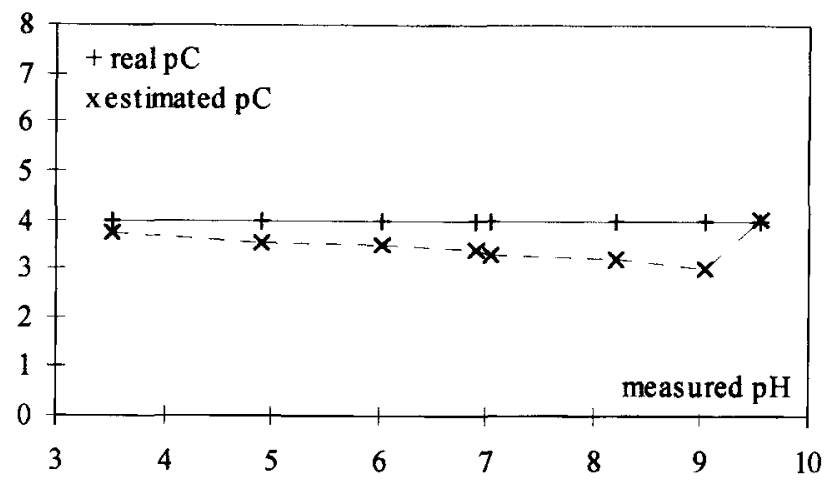

Figure $10 \quad \mathrm{pC}$ estimé dans le test 2. pC estimate, test 2.

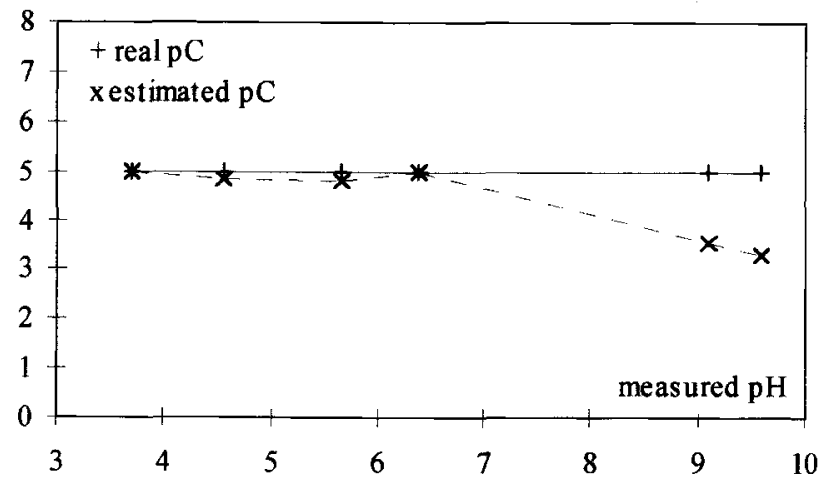

Figure $11 \quad p C$ estimé dans le test 3. $p C$ estimate, test 3. 


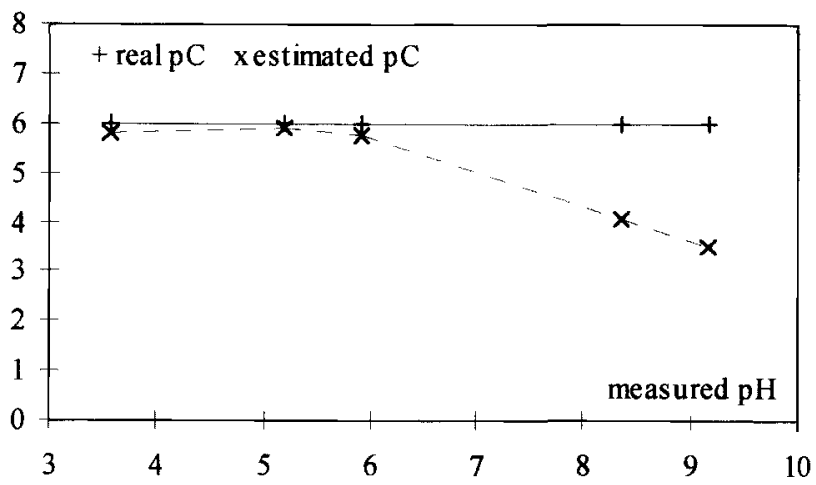

Figure $12 \quad \mathrm{pC}$ estimé dans le test 4.

$p C$ estimate, test 4.

À partir des résultats de ces tests nous reproduisons en figure 13 le domaine de validité d'une mesure de concentration de cuivre par ISE utilisant notre modèle.

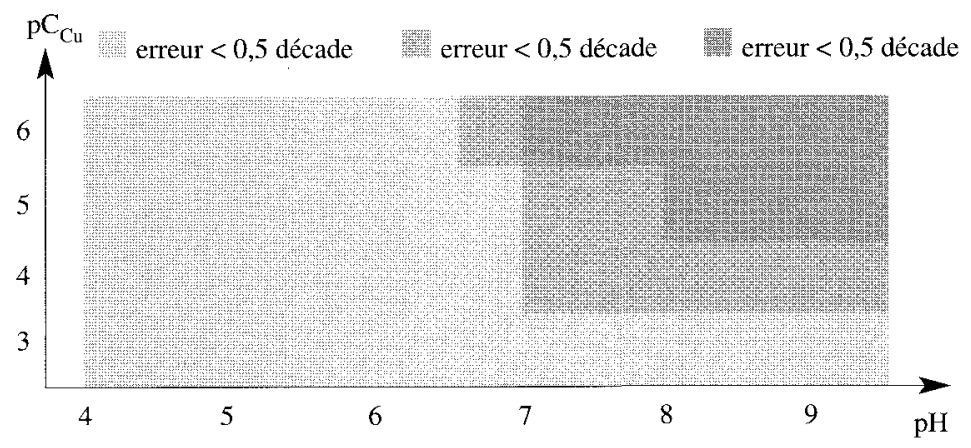

Figure 13 Erreur d'estimation.

Estimation error.

\section{6 - CONCLUSION}

Nous avons testé la faisabilité d'une mesure en continu de la concentration de cuivre dans une solution présentant des caractéristiques physicochimiques très variables principalement en terme de salinité et acidité.

Un banc de test destiné à étudier le comportement du dispositif de mesure est présenté. La reconstitution des conditions physicochimiques de milieux réels est obtenue par injection de produits chimiques dans la solution sous test. La réponse des capteurs est quasiment instantanée.

Le comportement de l'électrode spécifique ISE $(\mathrm{Cu})$ est modélisé par une loi non linéaire s'inspirant de la relation de Nernst généralisée. Le modèle prend 
en compte les paramètres : température, conductivité et $\mathrm{pH}$ du milieu. Le redox, fortement corrélé au $\mathrm{pH}$, est ici ignoré.

Nous avons testé les performances du dispositif en terme d'estimation de la teneur en cuivre. Celle ci peut être réalisée dans la gamme $10^{-6}$ à $10^{-3} \mathrm{~mol} / \mathrm{dm}^{3}$, pour un $\mathrm{pH}$ de 4 à 9 et une conductivité variable entre 400 et $2300 \mu \mathrm{s} \cdot \mathrm{cm}^{-1}$. La précision obtenue est d'autant meilleure que le taux de pollution par le cuivre est élevé. Les moins bons résultats sont relevés pour les faibles concentrations et lorsque le milieu est basique. Ils conduiraient, en situation réelle, à des fausses alarmes.

Finalement, bien que peu précise, la méthode proposée pourrait être utilisée comme un indicateur du niveau de concentration de cuivre dans les eaux de rejet.

La poursuite envisagée est une extension de cette méthode à la détection d'un groupe de métaux lourds. Dans cette perspective, nous devrons modifier le modèle afin de tenir compte des corrélations et des problèmes d'ions interférents ainsi que de l'influence de paramètre tel que le potentiel redox pour les valeurs faibles en dessous de $250 \mathrm{mV} / \mathrm{ENH}$ rencontrées fréquemment dans les eaux de rejet.

\section{RÉFÉRENCES BIBLIOGRAPHIQUES}

BOURGOGNON H., FOMBON J., LANCELOT F., PARIS J., ROUBIN M., TACUSSEL J., 1980. Étude et mise au point d'électrodes spécifiques à membrane solide polycristalline pour la détermination des ions cuivre. Analusis, 8, 7, 296-299.

CHARLOT G., 1969. Les réactions chimiques en solution : l'analyse qualitative minérale. Ed Masson.

COBBEN P.L.H.M., EGBERINK R.J.M., BOMER J., HAAK J.R., BERGVELD P., REINHOUDT D.N., 1992 Detection of heavy metal ions by ISFETs in a flow injection analysis cell.. Sensors and actuators B, 6, 304-307.

JUNG L., 1986. System identification, theory for the user, Prentice Hall Editions.

KATSUHITO H., HURT S., YONETANI A., HIRANO Y., 1994. Atomic Absorption Spectrophotometric System for Environment Water Analysis. Hitachi Review, 43, 4.

MIZIER M.O., 1995. La mesure en continu des rejets dans l'eau. Décision Environnement, $\mathrm{n}^{\circ} 37$.
SAES DE VITERI J., DERMOT D., 1994. Ammonium Detection Using an lon-Selcetive Electrode Array in Flow-Injection Analysis. Electroanalysis, 6, 9-16.

STELLA R., GANZERLI-VALANTINI M.T., 1980. The Use of Copper lon-Selective Electrode For Determination of Copper Chemical Forms in Natural Waters. Pergamon Ser. Environ. Sci., 3, 581-588.

TISSERAND P., 1998. Mesure en continu et in situ des pollutions dans les milieux acqueux : conception et réalisation d'une instrumentation adaptée à la détection de métaux lourds par potentiométrie sélective. Th. Doct. Univ. Nancy I, 187 p.

THOMAS M., FLORION A., CHRETHIEN D., TERVER D., 1996. Real-time biomonitoring of water contamination by cyanide based on analysis of the continuous electric signal emitted by a tropical fish: Apteronotus Albifrons. Wat. Res., 30 (12), 3083-3091. 